\title{
EXPLORANDO A AVALIAÇÃO: UM INSTRUMENTO BÁSICO PARA O GERENCIAMENTO DO CUIDADO EM SAÚDE COLETIVA
}

[Exploring assessment: a basic tool to care management in public health]

\author{
Olga Laura Giraldi Peterlini \\ Ivete Palmira Sanson Zagonel ${ }^{* *}$
}

RESUMO: O objetivo deste artigo é discutir os conceitos e abordagens metodológicas sobre avaliação e sua importância para o enfermeiro no exercício da gerência do cuidado. O enfermeiro em sua prática profissional cotidiana de gerenciamento do cuidado necessita de habilidades e conhecimentos que possibilitem $O$ desenvolvimento de ações administrativas. A avaliação é um instrumento administrativo utilizado sistematicamente pelo ser humano e tem sido apontada também como ferramenta necessária para o profissional enfermeiro na sua prática de cuidado. A importância da avaliação na área de saúde deve-se ao seu caráter processual, multidisciplinar e social, e situa-se na possibilidade de extrair subsídios para a tomada de decisão, de verificação da eficácia, da efetividade e da eficiência das ações de saúde desenvolvidas e na possibilidade de redirecionamento das mesmas para as reais necessidades da comunidade. Concluiu-se, portanto, que a avaliação possibilita um processo reflexivo da prática realizada e gera conseqüentemente um movimento de transformação da realidade e crescimento dos sujeitos envolvidos no processo na ação avaliada.

PALAVRAS-CHAVE: avaliação; avaliação em saúde; gerenciamento do cuidado.

\footnotetext{
*Enfermeira. Docente da Faculdade Evangélica do Paraná - FEPAR. Mestranda do Curso de Mestrado em Enfermagem da UFPR. Membro do Núcleo de Estudos, Pesquisa e Extensão em Cuidado Humano de Enfermagem - NEPECHE/UFPR.

"Enfermeira. Professora Sênior do Programa de Pós-Graduação em Enfermagem da UFPR. Doutora em Filosofia de Enfermagem.

Coordenadora do NEPECHE/UFPR. Coordenadora do Curso de

Enfermagem do Instituto de Ensino Superior Pequeno Príncipe - IESPP/ PR.
}

\section{INTRODUÇÃO}

$\mathrm{Na}$ área de saúde o enfermeiro é o profissional que cada vez mais vem sendo requisitado à desempenhar funções gerenciais em todos os níveis de atenção à saúde, principalmente na saúde coletiva (GIL, 1998).

$O$ enfermeiro na função gerencial enfrenta constantemente situações diversas e adversas que necessitam de tomada de decisão e esta, por sua vez, necessita de informações muitas vezes conseguidas por um processo de avaliação. Este processo na saúde se reveste de grande importância, quando consideradas que as ações de saúde podem afetar diretamente o homem.

A função gerencial exercida pelo enfermeiro é determinada historicamente, mas nos últimos anos, recebeu atenção especial devido a vários fatores. Entre estes se inclui a lei do exercício profissional, que no Art.11 estabelece como responsabilidade deste profissional 0 planejamento, a organização, a coordenação, a execução e avaliação dos serviços de assistência de enfermagem; as Diretrizes Curriculares Nacionais para os Cursos de Graduação em Enfermagem que recomendam em vários momentos no documento, o desenvolvimento da competência gerencial nos futuros profissionais enfermeiros; e a prática gerencial propriamente dita, no contexto atual do Sistema de Saúde de nosso país, já que a gerência tem sido colocada como uma ação estratégica, principalmente na perspectiva de viabilizar novos modelos de atenção à saúde.

Campos (2000) enfatiza a posição intermediária que o gerenciamento ocupa, ou seja, entre a definição de diretrizes políticas e a 
prestação direta de serviços, que confere à prática gerencial um espaço privilegiado, pois é neste espaço que são traduzidas as políticas e transformadas em formas concretas de organização e de ações de saúde.

Assim, o trabalho de gerência, por conviver com as distintas dimensões dos projetos de saúde, precisa dispor de instrumentos que lhe possibilitem dar sustentação e intervir adequadamente no plano técnico, ético, organizacional e político, que constituem esse trabalho. Um dos instrumentos apontados como necessários é a avaliação, em que Cianciarullo (2000) considera como instrumento básico e essencial para a prática profissional do enfermeiro. Entende-se por instrumentos, os recursos utilizados para se alcançar um objetivo ou conseguir determinado resultado.

Ela é uma ação envolvida com a vida: o ato de avaliar é ação imprescindível e está presente em várias situações cotidianamente. Segundo Gomes (1977), o homem, para desenvolver sua forma de sentir, pensar e agir necessita avaliar suas experiências como meio de identificar quais as suas possibilidades e restrições. Apesar de ser uma atividade do dia-a-dia e realizada como ação corriqueira, às vezes não intencional e não programada, percebe-se que a avaliação enquanto processo ainda não está incorporada na prática profissional do enfermeiro.

$A$ avaliação não se restringe a uma única dimensão; deve ser prevista não apenas para avaliar o próprio desempenho, como também para avaliar programas de saúde, metas institucionais e desempenho de outros profissionais.

Vários autores, como Donabedian (1990), Pereira (1995), Hartz (1997), Tanaka (2000, 2001), que têm trabalhado nas últimas décadas com avaliação em saúde, apresentam um consenso conceitual, uma vez que consideram que o ato de avaliar consiste fundamentalmente em fazer um julgamento de valor a respeito de uma intervenção/ ação ou sobre qualquer um de seus componentes, com o objetivo de ajudar na tomada de decisão e promoção de mudanças, visando tornar as ações mais racionais e efetivas.

A prática de avaliar, portanto, deve ser processual, sistematizada e contínua, e deve sempre ter por objetivo melhorar as atividades ainda em curso e ajudar no gerenciamento, no planejamento e replanejamento, e principalmente, na tomada de decisão. Diferente da avaliação da aprendizagem, as avaliações voltadas para as ações de saúde abordam rotineiramente dois caminhos: um é o da comparação e o outro é o de padrão. Comparar significa medir uma mesma situação em diferentes momentos e em diferentes cenários; e padrão refere-se a modelo, uma referência de uso conhecido e aceito pela comunidade cientifica (PEREIRA, 1995).

Reconhecendo a importância das questões abordadas acima, propomos neste artigo efetivar uma reflexão teórica sobre as questões conceituais e metodológicas relativas às ações de avaliação e sua importância no exercício do gerenciamento do cuidado pelo enfermeiro.

\section{POR QUE AVALIAR?}

A contribuição que a avaliação pode proporcionar é inestimável, não apenas no sentido de apontar acertos e ou falhas, mas principalmente por possibilitar o delineamento de soluções, reorganizar atividades e serviços, vislumbrando caminhos alternativos, com o intuito de maximizar a utilização de recursos disponíveis. Este aspecto tem sido relevante, principalmente em países de recursos financeiros escassos. O alto custo da atenção à saúde exige dos gestores decisões que beneficiem maior número de usuários e que consigam resultados mais eqüitativos com os mesmos recursos disponíveis (TANAKA, 2000).

Considera-se, portanto, que a ação de avaliação é instrumento capaz de nortear e apreender diversos momentos do processo de atenção à saúde, possibilitando, assim, entender melhor os sucessos e insucessos de suas práticas com o objetivo de promover, de forma contínua, a melhoria da qualidade dos serviços oferecidos com o menor custo financeiro possível.

O resultado de um determinado processo de avaliação tem sido recomendado para ser utilizado em função de uma melhor conscientização dos profissionais de saúde, e principalmente da própria comunidade, que assim passa a conhecer e refletir sobre seus problemas e necessidades.

Pereira (1995) vai além, porquanto considera que a ação de avaliação possibilita ao profissional uma atividade reflexiva questionadora não só da atividade ou ação em questão, mas também e 
principalmente do mundo sociocultural que o cerca. Esta possibilidade faz da avaliação uma atividade com característica transdisciplinar, pois necessita, na fase analítica da aplicação, de várias áreas do conhecimento: epidemiologia, administração, estatística, ciência social, ciência política, comunicação.

O processo de avaliação, portanto, permite que o enfermeiro dimensione em que grau os objetivos e metas foram atingidos; meça o impacto das ações na saúde da população; monitore eventos de saúde; identifique problemas espacialmente localizados; detecte aspectos positivos e negativos dos serviços; obtenha elementos que possam subsidiar o planejamento e a tomada de decisão; e oriente a distribuição de recursos humanos, materiais e financeiros. Assim, a avaliação constitui instrumento que não apenas torna possível o controle dos resultados, mas principalmente fornece subsídios necessários que permitem a realimentação do processo de gestão, criando nova dinâmica para a prática profissional do enfermeiro que, desse modo, justifica o redirecionamento do cuidado. Nessa perspectiva pode-se então considerar a avaliação no gerenciamento do cuidado como processo contínuo que deve permear todas as atividades de cuidado desenvolvidas, e é neste processo de avalia-analisa-recria que está a grandeza deste processo, que se concretiza na possibilidade de geração de novos conhecimentos.

\section{A ESCOLHA DO MÉTODO DE AVALIAÇÃO}

Existe um consenso de diversos autores e também da própria Organização Mundial da Saúde (OMS) em considerar a avaliação como processo, ou seja, ações organizadas e ordenadas, tendo começo, meio e fim, seqüenciais e sistemáticas, realizadas continuamente e necessitando para sua operacionalização dos seguintes passos: determinar e estabelecer finalidades e objetivos para aquela determinada avaliação; estabelecer para cada objetivo um padrão e a partir daí pensar ou selecionar um instrumento eficiente para o processo que seja aplicável ao estudo e a cada item considerado. Após a aplicação do instrumento, interpretar achados e anotar conclusões, utilizando sempre as informações obtidas para a melhoria dos programas, numa técnica de retroalimentação.

A avaliação de um programa se inicia com a definição de quais dimensões serão valorizadas, naquele processo específico, para se constituir nos parâmetros de referência, a partir dos quais serão construídos os critérios e indicadores a serem utilizados (LUCKESI, 2000).

Atualmente, várias propostas referentes à condução do processo de avaliação na área da saúde têm surgido. Uma das propostas que tem apresentado boa aceitação entre os pesquisadores é a elaborada por Donabedian (1990), que utiliza critério ou parâmetro nos processos de avaliação. O autor define critério como sendo algo que serve de norma, parâmetro, padrão, ou seja, um balizador para a avaliação, ou ainda um componente ou aspecto importante, relacionado com a estrutura do serviço ou do processo realizado, ou ainda com o resultado da assistência e que tenha relevância no concernente à qualidade da assistência prestada.

Estabelecendo uma forma tridimensional para a avaliação em saúde, Donabedian (1990) recomenda que para sua efetividade ela não pode ser restrita a uma só perspectiva ou a um determinado componente, mas que seja a integração de três componentes que permeiam as ações de saúde: estrutura, processo e resultado. Considerando que a avaliação deva sempre buscar os parâmetros de qualidade, a avaliação da estrutura, refere-se basicamente às condições das instalações físicas, equipamentos, móveis, materiais, insumos e recursos humanos, procurando sempre averiguar os aspectos qualitativos e quantitativos destes recursos disponíveis e necessários para o desenvolvimento de uma determinada ação, processo ou programa de saúde.

A avaliação do processo é orientada fundamentalmente para as ações das equipes de saúde e do cuidado com o indivíduo, família ou comunidade. Este aspecto é o mais difícil de ser avaliado, considerando-se que, no processo de cuidar, existem vários atores envolvidos e várias situações; portanto a tendência para avaliar esta dimensão é a utilização de protocolos, facilitando assim a ação comparativa dos processos. Neste caso, os protocolos seriam parâmetros, ou seja, a descrição de situações ou ações ideais que, comparados com o que está acontecendo com a 
realidade, indica se determinada atividade, processo ou ação se vêm adequando àquela situação especifica.

É importante considerar também um aspecto especial que ocorre no processo de cuidar, que é a interação com o paciente, apontada comumente como a responsável pelo sucesso das ações de saúde; e um aspecto muito difícil de ser verificado fora da ação, e que não há protocolo que dê conta e garanta a qualidade deste aspecto. Assim, o processo deve ser avaliado principalmente pela clientela, pois o sujeito da ação é o principal protagonista deste momento. Já a ação de avaliar o resultado, é mais objetiva e trata do estado de saúde do indivíduo ou de uma população como resultado de sua interação, ou ausência dela, com os serviços de saúde. Nesta dimensão, é possível utilizar indicadores, que permitem ações comparativas, principalmente no aspecto epidemiológico e evolutivo da saúde ou da doença. Outra questão inclusa nesta dimensão está relacionada com a satisfação do usuário, considerado por Peduzzi (2001) como o mais importante aspecto a ser avaliado, mesmo considerando que vários fatores podem afetar a percepção do paciente. É de extrema importância neste aspecto a opinião do cliente, alguns aspectos como acessibilidade, eqüidade, interação profissional /cliente e satisfação da expectativa, são considerados importantes indicadores de qualidade, e só poderão ser obtidos pela opinião do cliente (Donabedian, 1990).

Pereira (1995) considera que a avaliação das ações de saúde deve estar focada em três aspectos, que tem por objetivo verificar a eficácia e efetividade dos programas quando direcionadas para o resultado e para a eficiência quando se refere à alocação de recursos para a sua consecução. Um exemplo rotineiro acontece nas ações de imunização. Num primeiro momento se estabelecem metas referentes à ação de vacinação, ou seja, quantas vacinas vão ser necessárias e que deverão ser disponibilizadas de acordo com a meta estimada. Então se realizam as ações de administração das vacinas. A partir deste momento começa o processo de avaliação. A primeira avaliação refere-se ao aspecto quantitativo; é um processo comparativo entre o realizado com a meta estipulada; este é um dado que se refere à eficácia da ação, quanto mais o quantitativo da ação estiver mais próximo da meta estimada, mais eficaz foi a ação de vacinação. $A$ segunda avaliação é realizada algum tempo depois; esta tem por objetivo verificar a efetividade da ação, ou seja, se realmente a ação de imunização diminuiu consideravelmente a doença em exame. E a eficiência é verificada por um processo avaliativo que tem por objetivo mensurar o gasto realizado com o resultado obtido, ou seja, a relação de custo e beneficio da ação.

Tanaka (2001) propõe uma sistemática diferente na avaliação de serviços ou programas de saúde. A avaliação inicia-se pela análise do processo de trabalho e suas relações com os resultados, ou seja, com o estudo de impacto epidemiológico, visa entender as repercussões das ações operacionalizadas para atender a população usuária ou de referência. Essa característica metodológica da avaliação facilita o processo de utilização transdisciplinar de distintos conhecimentos existentes e disponíveis.

Especificamente para programas de saúde, Tanaka (2001) propõe que o processo de avaliação deva contemplar aspectos quantitativos e qualitativos. A avaliação quantitativa é basicamente utilizada quando se pretende conhecer a cobertura, a concentração e a eficiência de um programa; a avaliação qualitativa, quando se pretende personalizar, individualizar as ações de um determinado programa e conseqüentemente ir ao encontro da expectativa da clientela.

A idéia de qualidade está presente em todos os tipos de avaliação, uma vez que estes têm como característica o estabelecimento de um juízo, a atribuição de um valor a alguma coisa que, quando positiva, significa ter qualidade.

$\mathrm{O}$ ato de avaliar reflete a concepção que determinado gerente tem de um processo e o quanto ele deseja produzir transformações no âmbito de sua atuação. Também reflete o quanto ele se preocupa com a clientela assistida, pois a avaliação sempre deverá ser realizada tendo como beneficiário final o cliente do serviço/programa ou projeto (TANAKA, 2001). Portanto, a escolha do tipo de avaliação a ser adotada dependerá de quais dados o gerente necessita naquele momento e como ele vai utilizar tais dados. Observa-se que o método proposto por Pereira (1995) é indicado para medir o resultado. Se o dado final não é satisfatório 
ou ainda não está compatível com a meta estabelecida é que se buscará uma forma de verificar onde, em que momento a ação avaliada apresentou problema e o que interferiu no resultado final, ou seja, foi a estrutura ou o processo necessário a um determinado cuidado profissional.

Muitas ações do cuidado de enfermagem são ações técnicas, e essas não podem ser avaliadas somente no seu resultado. Porquanto, caso a avaliação indicar algum dado não satisfatório, o processo já foi realizado e o prejuízo contabilizado. Essa é a diferença entre avaliar ações ditas sociais e outras ações onde o ser humano é cliente envolvido apenas indiretamente. Neste caso, o ser humano é cliente e produto ao mesmo tempo. Caso o processo não tenha sido bem feito, trará conseqüências muitas vezes imediatas e irrecuperáveis ao paciente.

Esta proposta de avaliação deve começar com a escolha dos critérios, passar pelo compartilhamento das informações pelos parceiros formuladores e gestores e por momentos de discussão e publicização dos resultados. Ao serem divulgados de forma regular, os dados possibilitam que todos os envolvidos possam refletir sobre as mudanças e o que elas provocaram na vida da comunidade.

Tem sido proposta a utilização de outros sujeitos no processo de avaliação que não o usuário direto, principalmente quando se pretende medir o impacto de uma determinada ação ou programa, ou ainda quando o processo de avaliação requeira promover uma ação reflexiva com todos os sujeitos. Essa perspectiva é enriquecedora, porque possibilita que a avaliação seja realizada sob vários pontos de vista. Este processo é chamado de avaliação participativa (BASTOS, 2000).

Voltando ao exemplo das ações de imunização, determinada campanha poderá optar por avaliar também qualitativamente suas ações e incluir o parecer de clientes indiretos, como os pais, o funcionário que separou e distribuiu as vacinas, os funcionários da própria unidade de saúde. Assim, o processo de avaliação apresentará resultados mais adequados para uma ação executada diretamente com pessoas e para pessoas.

$\mathrm{Na}$ realidade brasileira, a incorporação da avaliação na gestão, nos serviços e programas, ainda é incipiente e assistemática. Assim, pode ser mais fácil iniciar a avaliação por um enfoque quantitativo. Com o sistema de informação implantado pelo Ministério da Saúde na última década, é possível acessar informações sobre mortalidade (SIM), nascimentos (SINASC), assistência hospitalar e ambulatorial (SIH e SIA), assistência básica (SIAB), avaliação nutricional (SINAN), dados de Programas como da Assistência ao Pré-Natal e Nascimento (SISPRÉNATAL) e câncer do colo de útero (SISCOLO).

Nesta direção, pode-se iniciar uma avaliação centrada na produção de serviços e ações, por meio da análise de cobertura, de produção e produtividade, e a partir daí, relacionar esses resultados com a estrutura existente e o processo realizado.

Em geral, as situações avaliadas no campo da saúde irão exigir a adoção de múltiplas abordagens, para que não se perca a capacidade de explicação e compreensão da situação. A avaliação quantitativa e qualitativa sistemática das ações programadas é uma das recomendações da Norma Operacional de Assistência à Saúde (NOAS) que, por meio de pactuação de indicadores no nível: federal, estadual e municipal, obriga os gestores a estabelecerem processos sistemáticos de acompanhamento dos indicadores pactuados (BRASIL, 2001).

Quando a prática avaliativa buscar compreender com maior profundidade 0 significado das relações entre as ações e os resultados, este caminho exige abordagem com predominância qualitativa.

A dificuldade reside justamente em decidir, objetivamente, quais são os parâmetros que expressam qualidade e como medi-los adequadamente. A existência de muitos critérios a serem considerados na avaliação da qualidade, tem o importante papel de realçar que a qualidade não pode ser traduzida por apenas um deles. A eficácia, a efetividade e a eficiência têm recebido particular atenção, outros como a equidade, aceitabilidade, a acessibilidade, a adequação e a qualidade técnico-científica, a continuidade, a coordenação do cuidado e o relacionamento do profissional de saúde com o paciente são critérios que poderão ser escolhidos como indicadores de qualidade de um determinado serviço ou 
procedimento (NOVAES, 1996).

Segundo Donabedian (1990), a avaliação da qualidade é constituída por dois componentes principais: o técnico-científico e a relação interpessoal. O componente técnico-científico diz respeito à precisão do diagnóstico, à terapêutica prescrita, à resolutividade, ou seja, ao aspecto técnico do cuidado. Já a relação interpessoal, embora subjetiva, refere-se à abordagem profissional-cliente e pode interferir no desfecho do cuidado prestado.

\section{GERENCIANDO O CUIDADO EM SAÚDE COLETIVA}

É inegável que o processo de avaliação se tornou uma necessidade e uma exigência contemporâneas. São inúmeras as justificativas citadas no início deste texto, mas há duas razões que devem demandar uma reflexão mais profunda nos profissionais enfermeiros. Uma refere-se à função gerencial assumida por este profissional especificamente na área da saúde coletiva, que não é somente função gerenciadora do cuidado prestado pela equipe de enfermagem, mas das ações desenvolvidas pelas unidades de saúde, ou dos distritos sanitários, ou ainda dos centros de especialidades e de hospitais comunitários, envolvendo práticas profissionais distintas, que tem por objetivo desenvolver o cuidado para uma parcela maior da população.

Outra questão que justifica sobremaneira a necessidade de um processo de avaliação sistematizado é a tendência de cada dia mais, as intervenções terem como princípio a participação da comunidade na condução das políticas de saúde e a incorporação do espírito da qualidade pela comunidade não só usuária do sistema, mas também pelos trabalhadores do setor.

A saúde é a política que mais avançou na construção de um novo projeto, pois foi formulada tendo como base a descentralização das ações de saúde e do planejamento integrado e participativo, mas mesmo assim, necessita mudar práticas e valores e introduzir outros instrumentos de gestão que possibilitem sair do discurso e caminhar para o seu efetivo reconhecimento. Duro (1998) e Berto (2000) indicam que o principal problema é a pouca utilização das informações em saúde no processo decisório na gestão do Sistema
Único de Saúde (SUS), indicando uma desarticulação dos processos de planejamento e de gestão com os de avaliação e monitoramento dos indicadores de saúde disponíveis.

Nesta especificidade é que o processo de avaliação ganha maior importância, pois poderá fazer a ponte, a ligação entre o processo de planejamento e o processo de gestão. Portanto, a avaliação deve permear todos os programas e ações ofertadas pela instituição de saúde. Daí, a necessidade de que a avaliação seja compartilhada por todos os profissionais e usuários, a fim de que constitua um momento de reflexão e de mobilização para mudanças na realidade da saúde e dos serviços (TAHARA, 1997).

O processo de avaliação sistemático exige não só maior capacidade técnica como também o desenvolvimento de capacidades sociais, para responder a um papel ainda novo no contexto brasileiro que é o de possibilitar a participação da comunidade.

É fundamental que o enfermeiro, ao gerenciar o cuidado, conheça a situação atual dos Sistemas de Informação em Saúde (SIS), seus dados e indicadores existentes e disponíveis na base federal, estadual e municipal e procure institucionalizar e socializar os mesmos para a comunidade, utilizando-o como instrumento de direcionamento das ações de cuidado de saúde no seu município ou na sua área de atuação.

Hoje dispõe-se de um conjunto de indicadores que são monitorados e disponibilizados pela Rede Integrada de Informações para a Saúde (RIPSA), coordenada pelo Ministério da Saúde e integrada por diversas instituições, com o apoio da Organização Panamericana de Saúde (OPAS). Esses indicadores estão agrupados em demográficos, socioeconômicos, de morbidade e fatores de risco, recursos e coberturas. As Secretarias Estaduais de Saúde já disponibilizam tais indicadores com abrangência estadual e municipal. Disponibilizam ainda, os dados e indicadores gerados pelos sistemas de informações dos programas específicos, como o Programa de Detecção Precoce do Câncer de Colo do Útero e o Programa de Humanização do Pré-Natal e Nascimento já implantados em todos os municípios do Paraná desde 2000.

O profissional enfermeiro assume atividades 
gerenciais do cuidado em todos os campos de atuação e deve conscientizar-se que esta atividade exige a utilização de diversos instrumentos de gestão e que o processo de avaliação é um instrumento utilizado como prática sistematizada, visando ao direcionamento e redirecionamento das ações. A proposta de experimentar uma abordagem transdisciplinar e participativa direciona a avaliação em saúde de modo que esta contribua para o desenvolvimento de uma competência coletiva, fundamental para a reorganização e produção de ações e serviços de saúde à altura das necessidades da população.

\section{CONSIDERAÇÕES FINAIS}

As transformações na área da saúde coletiva nos últimos anos, principalmente em face da implantação de novas propostas de modelo assistencial, de gerenciamento e da participação efetiva da comunidade, têm exigido dos profissionais formadores de recursos humanos, 0 acompanhamento e a reflexão no sentido de buscar métodos e estratégias que propiciem a aprendizagem necessária aos futuros profissionais enfermeiros. Aprendizagem que possibilite na prática profissional desenvolver ações que promovam respostas imediatas e concretas às reais necessidades da comunidade. Esta preocupação nos levou a buscar na literatura o tema avaliação, considerando-o como elemento e instrumento essencial para a prática do gerenciamento do cuidado e para as ações de gerenciamento que o enfermeiro assume cada vez mais na área da saúde coletiva.

Concluiu-se, portanto, que a avaliação deve ser um processo e uma ação sistematizada no cotidiano da prática do enfermeiro, que pode ser uma ação solitária ou contar com a participação dos sujeitos envolvidos na ação avaliada. Esta última, a mais rica, promove a participação e a coresponsabilidade, gera conhecimentos e redireciona as ações para as reais necessidades dos sujeitos envolvidos.

Quem introduz o processo de avaliação na sua rotina, demonstra responsabilidade e compromisso com a transformação, pois a avaliação gera a inquietação, a reflexão e a busca pelo melhor, isto é, pela qualidade e pela própria excelência.

A avaliação é uma instância formadora por excelência, cujo principal propósito é permitir melhorar, corrigir erros e deficiências, assinalando, passo a passo, o caminho para a tomada de decisão. Enquanto planejar significa um olhar sobre o futuro, iluminando a ação presente, a avaliação possibilita o redirecionamento da ação presente com vistas ao resultado futuro.

ABSTRACT: The objective of this article is to discuss methodological concepts and approaches related to assessment in the exercise of nursing care management. In daily practice of care management, nurses should have abilities and knowledge to carry out planning, organization, coordination, execution and assessment actions. Assessment is a life-involving endeavor which is present in many daily nursing practices. The importance of evaluation in the field of health is due to its process, multifaceted, and social character. Evaluation makes possible to obtain grounded information for the decision making process, for the assessment of efficacy, effectiveness, and efficiency of health actions as well as for reorientation of these actions towards the actual needs of the communities. Thus, evaluation allows for a reflective process of praxis and generates both changes in the real world and growth of the individuals involved in the action that was evaluated.

KEY WORDS: assessment, health evaluation, care management.

\section{REFERÊNCIAS}

BASTOS. A.C. Privilegiando a percepção do usuário na avaliação em saúde. Salvador, In: VI Congresso Brasileiro de Saúde Coletiva; 2000, Salvador. Relação de trabalhos. Salvador: 2000. $1 \mathrm{CD}-\mathrm{ROM}$.

BERTO, G. S. A participação do enfermeiro no processo decisório. Florianópolis, 114 p., 2000. Dissertação (Mestrado), Universidade Federal de Santa Catarina.

BRASIL. Ministério da Saúde. Portaria 95, de 26/ 1/01: aprova a NOAS_SUS 01/2001. Diário Oficial da União, Brasília, MS, 26 jan 2001.

CAMPOS. F.E. Avaliação da satisfação dos 
consumidores e dos profissionais de saúde com os serviços de saúde. Uma abordagem qualitativa. Salvador, In: VI Congresso Brasileiro de Saúde Coletiva; 2000, Salvador. Relação de trabalhos. Salvador: 2000. 1 CD-ROM.

CIANCIARULLO, T. I., ZANEI, S. S. V. et al. Instrumentos básicos para o cuidar: um desafio para a qualidade de assistência. São Paulo: Atheneu, 2000.

DONABEDIAN, A. Garantía y monitoria de la calidad de la atención médica: un texto introdutorio. México: Instituto Nacional de Salud Pública, DF, 1990.

DURO, J. A.Z. Decidir ou não decidir: o processo decisório. Rio de Janeiro: Qualitymark,1998.

GIL, C.R.R. Desenvolvimento gerencial nas Unidades Básicas de saúde: Uma oportunidade para a melhoria da gestão pública.In: Congresso Brasileiro de Enfermagem, 50², 1998, Salvador.

Anais... Salvador: Associação Brasileira de Enfermagem-seção Bahia, 1998. p. 184-191.

GOMES, D.L.S. Avaliação em Saúde. Ribeirão Preto, 1977. 166 p. Dissertação (Mestrado em Enfermagem) - Escola de Enfermagem, Universidade de São Paulo.

HARTZ, Z.M. A., organizadora. Avaliação em saúde: dos modelos conceituais à prática na análise da implementação de programas. Rio de Janeiro: Fiocruz, 1997.

LUCKESI, P.T.R. A avaliação da Gestão descentralizada do SUS no município uma proposta metodológica. In: VI Congresso Brasileiro de Saúde Coletiva; 2000, Salvador. Relação de trabalhos. Salvador: 2000. 1 CD-ROM

NOVAES, H. M.D. Epidemiologia e avaliação em serviços de atenção médica: novas tendências na pesquisa. Caderno de Saúde Pública. n.2, p. 712,1996 .

ORGANIZAÇÃO MUNDIAL DE LA SALUD. Administração dos serviços de higiene materno-infantil. Serv. Inf. , (115), 1957 Apud Gomes DLS. Avaliação em saúde. Ribeirão Preto,
1977. 166p. Dissertação de Mestrado - Escola de Enfermagem, Universidade de São Paulo.

PEDUZZI, M. Laços e compromissos e contradições existentes nas relações de trabalho de enfermagem. In: $53^{\circ}$ CONGRESSO BRASILEIRO DE ENFERMAGEM. Curitiba, 2001. Anais...Curitiba: ABEn-PR, 2001. p.167- 182

PEREIRA, Maurício Gomes. Epidemiologia: teoria e prática. Rio de Janeiro: Guanabara Koogan, 1995.

TAHARA, Angela Tamiko et al. Satisfação do paciente: refinamento de instrumento de avaliação de serviços de saúde. Revista Brasileira de Enfermagem, v.50, n.4, p.497-506, out/dez,1997.

TANAKA, O. Y. MELO, C. M. Uma proposta de abordagem transdisciplinar para avaliação em saúde. Salvador. In: VI Congresso Brasileiro de Saúde Coletiva; 2000, Salvador. Relação de trabalhos. Salvador: 2000. 1 CD-ROM

. Avaliação de programas de saúde do adolescente: um modo de fazer. São Paulo: EdUSP, 2001.

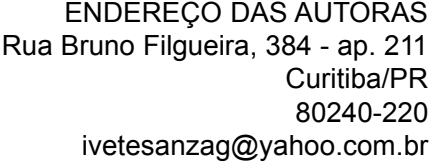

ivetesanzag@yahoo.com.br 